\title{
Optimization of a traveling wave superconducting rf cavity for upgrading the International Linear Collider
}

\author{
V. Shemelin®, H. Padamsee, and V. Yakovlev® \\ FNAL, Batavia, Illinois 60510, USA
}

(Received 10 October 2021; accepted 24 January 2022; published 7 February 2022)

\begin{abstract}
The standing wave TESLA niobium-based superconducting radio frequency structure is limited to an accelerating gradient of about $50 \mathrm{MV} / \mathrm{m}$ by the critical rf magnetic field. To break through this barrier, we explore the option of niobium-based traveling wave (TW) structures. Optimization of TW structures was done considering experimentally known limiting electric and magnetic fields. It is shown that a TW structure can have an accelerating gradient above $70 \mathrm{MeV} / \mathrm{m}$ that is about 1.5 times higher than contemporary standing wave structures with the same critical magnetic field. The other benefit of TW structures shown is $R / Q$ about 2 times higher than the TESLA structure that reduces the dynamic heat load by a factor of 2 . A method is proposed how to make TW structures multipactor-free. Some design proposals are offered to facilitate fabrication. Further increase of the real-estate gradient (equivalent to $80 \mathrm{MV} / \mathrm{m}$ active gradient) is also possible by increasing the length of the accelerating structure because of higher group velocity and cell-to-cell coupling. Realization of this work opens paths to International Linear Collider energy upgrades beyond 1 to $3 \mathrm{TeV}$ in competition with CLIC. The paper will discuss corresponding opportunities and challenges.
\end{abstract}

DOI: 10.1103/PhysRevAccelBeams.25.021001

\section{INTRODUCTION}

A strong physics attraction for the International Linear Collider (ILC)—besides the Higgs and Top Factories [1-2] -is the inherent energy upgradability. As described in the ILC technical design report [3], ILC offers paths to energy upgrades of 0.5 and $1 \mathrm{TeV}$ for which higher gradients are critical for affordability, as cavities and cryomodules are dominant cost drivers. There has been steady progress in single-cell and multicell cavity gradients [4] over the past 3+ decades along with SRF science and technology advances. Proof of principle is already in hand for cavity preparations that deliver single-cell TESLA-shape cavities with gradients up to $49 \mathrm{MV} / \mathrm{m}$ [5-6], and for nine-cell cavities with gradients up to $45 \mathrm{MV} / \mathrm{m}$ [7]. These gradient advances come from high purity, high residual resistance ratio $\mathrm{Nb}$, electropolishing at low temperatures, and optimized $120^{\circ} \mathrm{C}$ baking in two steps, $800^{\circ} \mathrm{C}$ furnace treatment for hydrogen removal, and about 100 bar high pressure water rinsing for removal of field emission particulates. The fundamental critical magnetic field of approximately $210 \mathrm{mT}$ presents the ultimate hard limit to niobium cavity gradients. For the standing wave TESLA shape structure, with peak surface

Published by the American Physical Society under the terms of the Creative Commons Attribution 4.0 International license. Further distribution of this work must maintain attribution to the author(s) and the published article's title, journal citation, and DOI. magnetic field to accelerating field ratio $B_{\mathrm{pk}} / E_{\mathrm{acc}}=$ $4.26 \mathrm{mT} /(\mathrm{MV} / \mathrm{m})$, this limit translates to a maximum gradient of $50 \mathrm{MV} / \mathrm{m}$. Fundamental limits for field emission linked to high peak electric field are presently not known and have so far not been encountered for surface fields. The practical—not fundamental-limit in principle can be overcome with technology advances in surface preparation (such as more effective final high-pressure rinsing). Further gradient advances to $50-59 \mathrm{MV} / \mathrm{m}$ with the surface electric field up to $125 \mathrm{MV} / \mathrm{m}$ have been demonstrated [8-10] in single-cell cavities of advanced geometries with $10 \%-15 \%$ lower $B_{\mathrm{pk}} / E_{\mathrm{acc}}$, such as reentrant, low loss, Ichiro, and low surface fields shapes. Progress toward attainment of $50 \mathrm{MV} / \mathrm{m}$ accelerating rate in multicell standing wave niobium cavity was shown recently [11].

Even higher gradients are needed for ILC energy upgrades beyond $1 \mathrm{TeV}$. This paper discusses optimized traveling wave (TW) superconducting niobium-based structures [12] with effective gradients up to $73 \mathrm{MV} / \mathrm{m}$ to open upgrade paths to $3 \mathrm{TeV}$, in competition with CLIC at $3 \mathrm{TeV}$. It is suggested in $[13,14]$ that the overall cost for $3 \mathrm{TeV}$ ILC with $70 \mathrm{MV} / \mathrm{m}$ gradient is comparable to CLIC $3 \mathrm{TeV}$, and the ac power is $190 \mathrm{MW}$ lower. TW structures offer two main advantages compared to standing wave (SW) structures: substantially lower peak magnetic and peak electric field ratios, and substantially higher $R / Q$ (for lower cryogenic losses, and lower if power demand). In addition, TW structure operates far off the passband boundaries, and therefore, has high stability of the field 


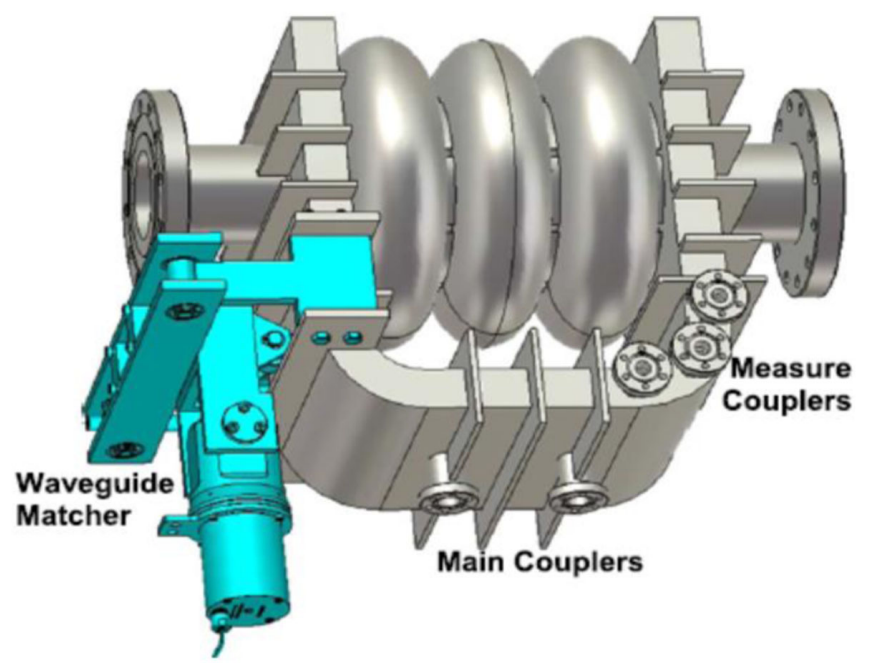

FIG. 1. Three-cell unit prepared at Fermilab by Euclid Techlab.

distribution along the structure with respect to geometrical perturbations [12]. This allows a much longer structure length and hence no gap between short (1 meter) cavities, thereby increasing the real-estate gradient, but this advantage substantially increases the engineering challenges. Besides, the TW structure requires a feedback waveguide for redirecting power from the end of the structure back to the front to avoid high peak surface fields in the accelerating cells. This requires careful tuning to compensate reflections along the TW ring to obtain a pure traveling wave regime at the desired frequency. Because the beam bunch charge for the $3 \mathrm{TeV}$ upgrade is 3 times lower than the bunch charge for $0.5 \mathrm{TeV}$ [13], (for lower IP backgrounds) it is further possible to lower the aperture (from 70 to $50 \mathrm{~mm}$ ) to obtain an overall $50 \%$ reduction in $B_{\mathrm{pk}} / E_{\text {acc }}$ and factor of 2 gain in $R / Q$ over the TESLA standing wave structure. The lower bunch charge reduces the wakefields.

Previously, substantial progress was made at Fermilab and in Euclid Techlab on the way to realization of the TW structure in the regime of a resonant ring [15-17]. A onecell cavity prepared by this joint team reached $26 \mathrm{MV} / \mathrm{m}$ with interior (easier) treatment of BCP (Buffered Chemical Polishing). It should be better with EP/baking (EP stands for Electropolishing). The single-cell cavity was not tested in the traveling wave regime. The waveguide in this experiment was used for demonstration of (i) a possibility of its production, (ii) effective cleaning without deterioration of the $Q$ factor. A three-cell cavity, Fig. 1, is now ready for test at Fermilab. The entire cavity-periodic structure, waveguide, transitions-is welded together [17]. It is supposed that initially BCP is used to process the periodic structure and the feedback waveguide, and then EP is applied for the structure to increase the acceleration gradient, BCP is expected to be sufficient for the waveguide because fields in the waveguide are much smaller than in the structure.
Technology of the TW SRF cavity-mechanical or electromagnetic design, manufacturing and processingis not the subject of the paper. Nevertheless, a couple of technological solutions are offered, because they can help to solve a problem of narrow gaps between adjacent cells, and further increase achievable surface electric field - this is a solid iris disk and a possibility of diamond turning, successfully used for normal conducting cavities.

The subject of the paper is optimization of the cell of the periodic acceleration structure to demonstrate TW SRF cavity potential. In addition to periodic structure discussed in the paper, the TW SRF structure has the following components: feedback waveguide (WG); transition from WG to the periodic structure; matcher in the waveguide necessary for internal reflection compensation to maintain pure TW; high-power coupler necessary for TW excitation; diagnostic couplers in the waveguide; tuner; He vessel.

Most of these elements are described in detail in a row of papers; $[12,15,16,17]$ and others.

The optimizations described below are enabled by accurate calculations of cavity parameters. 2D computer code SuperLANS [18], used for this purpose, has the accuracy necessary for these optimizations.

\section{GEOMETRY OF AN ELLIPTICAL CAVITY}

Contemporary superconducting rf cavities for high energy particle accelerators consist of a row of cells coupled together as shown in Fig. 2(a). The contour of a half cell consists of two elliptic arcs and a straight segment tangential to both. The contour can be described by several geometrical parameters shown in Fig. 2(b). Three of these parameters, length of the half-cell $L$, aperture $R_{a}$, and equatorial radius $R_{\text {eq }}$ are defined by physical requirements: in the case of a traveling wave $L=\theta \lambda /(2 \pi)$, where $\theta$ is the phase advance per cell, and $\lambda$ is the wavelength; the aperture is defined by requirements for coupling between cells and by the level of wakefields that can be allowed for a given accelerator; and the equatorial radius $R_{\mathrm{eq}}$ is used for tuning the cavity to a given frequency. The remaining four parameters $(A, B, a, b)$ can fully describe the geometry. Here $A, B$ and $a, b$ are the half axes of the equatorial and iris constitutive ellipses, respectively. The best combination of four parameters is the goal for the cavity shape optimization. The angle of the wall inclination between the axis of rotation and the straight segment of the wall is designated as $\alpha$. The cavity with $\alpha<90^{\circ}$ is known as the reentrant cavity.

\section{CELLS WITH DIFFERENT $L-A$}

Theoretically, the value of $A$ [see Fig. 2(b)] is limited by the value of $L$ though there is a technological limit for the gap between outer surfaces of cells, $2(L-A-t)$, defined by the thickness $t$ of material and needed for welding two half cells together. 


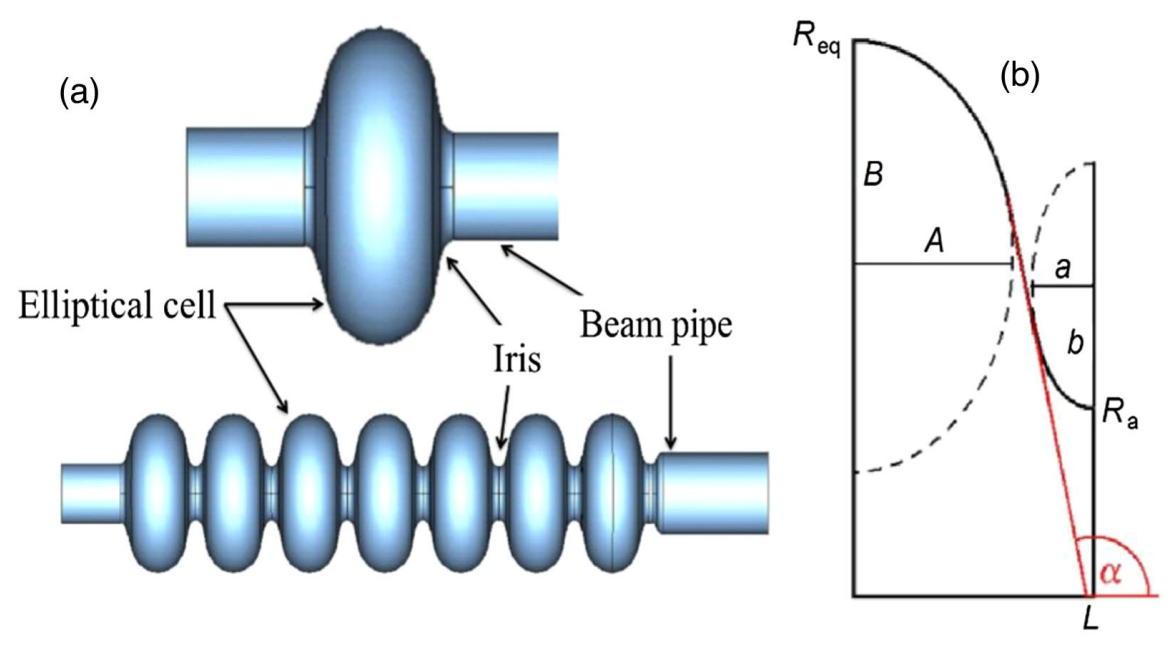

FIG. 2. (a) Single cell and multicell elliptical cavities; (b) geometry of the half cell.

To investigate the whole set of parameters, we will optimize the cavity with $E_{\mathrm{pk}} / E_{\mathrm{acc}}=2$ for different values of $L-A$. Here, the phase advance angle $\theta=105^{\circ}$ and the aperture radius $R_{a}=30 \mathrm{~mm}$. The results for $L-A=0,1$, and 5 are presented in Table I.

One can see from the Table that benefits of the reentrant shape, lower $B_{\mathrm{pk}} / E_{\mathrm{acc}}$ and higher $R_{\mathrm{sh}} / Q$, cannot be realized for the realistic cavity with $\theta=105^{\circ}$ because of shorter cell length than in the $180^{\circ}$ standing wave cavity, and the necessity to have a nonzero thickness of material and the gap for welding are more crucial than for the $180^{\circ}$ cavity.

A discussion with an expert [19] revealed that even for the value of $L-A=5 \mathrm{~mm}$ there may be a problem for mass production to use a stiffening ring between the cells to make the cavity more robust. However, for the three-cell TW cavity [17] a unique welding technology has been developed by Advanced Energy Systems, which allowed to succeed in installation of the stiffening ring in the gap $L-A$ of $5.1 \mathrm{~mm}$, see Fig. 3. The diameter of the stiffening ring is selected as a compromise between the cavity tunability, and Lorentz force detune, it is $130 \mathrm{~mm}$.

TABLE I. Parameters of optimized cells for different values of $L-A . E_{\mathrm{pk}} / E_{\mathrm{acc}}=2$, phase advance per cell $\theta=105^{\circ}$, aperture $2 R_{a}=60 \mathrm{~mm}$.

\begin{tabular}{lccc}
\hline \hline$L-A, \mathrm{~mm}$ & 0 & 1 & 5 \\
$A, \mathrm{~mm}$ & 33.631 & 32.631 & 28.631 \\
$B, \mathrm{~mm}$ & 34.294 & 37.304 & 38.919 \\
$a, \mathrm{~mm}$ & 5.284 & 5.233 & 4.903 \\
$b, \mathrm{~mm}$ & 7.777 & 7.680 & 6.790 \\
$B_{\mathrm{pk}} / E_{\mathrm{acc}}, \mathrm{mT} /(\mathrm{MV} / \mathrm{m})$ & 2.848 & 2.833 & 3.027 \\
$R_{\mathrm{sh}} / Q, \mathrm{Ohm} / \mathrm{m}$ & 1995 & 1967 & 1820 \\
$\alpha$, degrees & 71.81 & 73.26 & 90.23 \\
$R_{\mathrm{eq}}, \mathrm{mm}$ & 95.526 & 96.919 & 100.255 \\
\hline \hline
\end{tabular}

As can be seen in this figure, not only welding of cells is hampered in a narrow but the insertion of a stiffening ring also becomes complicated.

A possible solution of this problem can be changing the cavity design, see Fig. 4. Here, instead of two half cells welded together, the half cells are welded to an iris disk. The place of the weld from the inner side of the cells can be taken at the circle where the electric and magnetic fields are in a balance $\varepsilon_{0} E^{2}=\mu_{0} H^{2}$. In this case, both fields are much

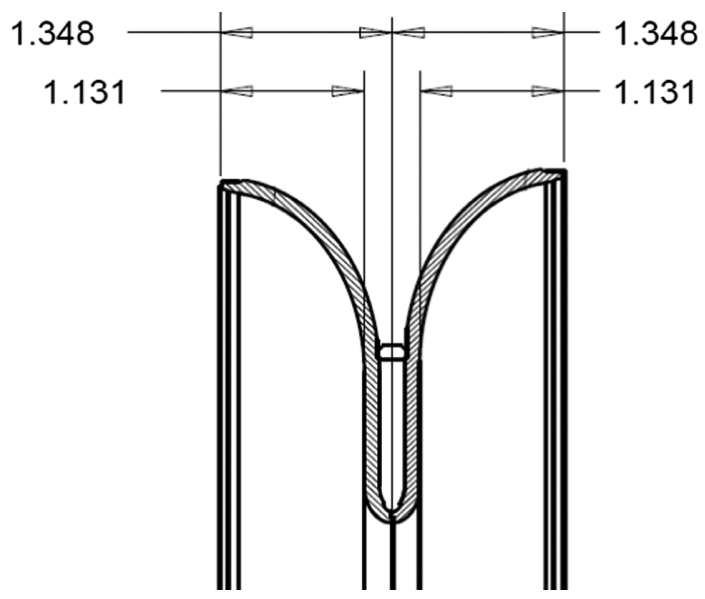

FIG. 3. An example of cavity with $L-A=5.5 \mathrm{~mm}$.
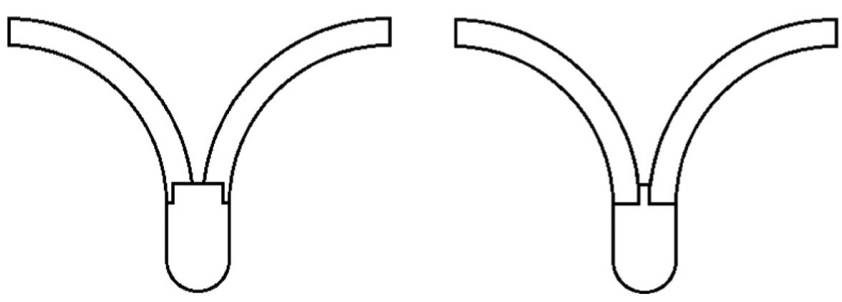

FIG. 4. New possible design of the cells' connection. 
lower than at their maxima, and a small perturbation by the welding bead will not change the frequency and other important figures of merit. This design makes it possible to get rid of the stiffening rings because the iris disk is stiff. Somewhat increased distance from the iris tip to the cooling agent is not important because heat production by the surface rf current in this area is negligible. (Risk of loss of the superconductivity due to heat production by the field emission or by the heat deposition from intercepted energetic electrons persists like in a traditional design and should be evaluated.) The iris tip can be made of any shape in accordance with optimization. The radius of curvature at the iris tip becomes comparable to the thickness of the niobium sheet when the shape is optimized, which makes it difficult to make it by deep drawing. In the case of a solid disk, this problem is removed.

For production of the iris disk, the experience of production TW X-band normal conducting accelerating structures can be used [20]. This structure reached the accelerating gradient up to $100 \mathrm{MeV} / \mathrm{m}$ that was due not only to higher frequency but also to diamond turning which gives very high smoothness of surface and precise dimensions as compared to deep drawing. Cavities machined out of bulk niobium are expensive [21], but the iris disk for a TW cavity will be much thinner and smaller in diameter than we would use to make a whole SW cavity. The combination of diamond turning, and chemical treatment and rinsing used for SW cavities can further improve the surface quality needed for suppression of field emission. In optimization, the higher is $E_{\mathrm{pk}} / E_{\mathrm{acc}}$, the lower $B_{\mathrm{pk}} / E_{\mathrm{acc}}$ can be reached as it was shown in [22].

\section{CELLS WITH DIFFERENT APERTURES $\boldsymbol{2}_{a}$}

A smaller aperture dramatically increases the accelerating rate because of smaller $B_{\mathrm{pk}} / E_{\mathrm{acc}}$ for the same value of $E_{\mathrm{pk}} / E_{\mathrm{acc}}$ and, hence lower probability of magnetic quench. This decrease of $B_{\mathrm{pk}} / E_{\mathrm{acc}}$ together with an increase of $R_{\mathrm{sh}} / Q$ vs a decrease of $R_{a}$ is illustrated in Table II.

TABLE II. Parameters of optimized cells for different values of aperture radius $R_{a} . E_{\mathrm{pk}} / E_{\mathrm{acc}}=2$, phase advance per cell $\theta=105^{\circ}$, aperture $2 R_{a}=60 \mathrm{~mm}$.

\begin{tabular}{lccc}
\hline \hline$R \mathrm{a}, \mathrm{mm}$ & 35 & 30 & 25 \\
$A, \mathrm{~mm}$ & 28.631 & 28.631 & 28.631 \\
$B, \mathrm{~mm}$ & 39 & 38.919 & 36.986 \\
$a, \mathrm{~mm}$ & 6.2 & 4.903 & 3.872 \\
$b, \mathrm{~mm}$ & 8.7 & 6.790 & 5.159 \\
$B_{\mathrm{pk}} / E_{\mathrm{acc}}, \mathrm{mT} /(\mathrm{MV} / \mathrm{m})$ & 3.219 & 3.027 & 2.858 \\
$R_{\mathrm{sh}} / Q, \mathrm{Ohm} / \mathrm{m}$ & 1607 & 1820 & 2.048 \\
$\alpha$, degrees & 85.77 & 90.23 & 91.98 \\
$R_{\mathrm{eq}}, \mathrm{mm}$ & 101.333 & 100.255 & 99.035 \\
\hline \hline
\end{tabular}

\section{NEW APPROACH FOR OPTIMIZATION}

Optimization of an elliptical cavity is usually done as a search for minimum $B_{\mathrm{pk}} / E_{\mathrm{acc}}$ when the value of $E_{\mathrm{pk}} / E_{\mathrm{acc}}$ is given. It is also possible to minimize $E_{\mathrm{pk}} / E_{\mathrm{acc}}$ for a given $B_{\mathrm{pk}} / E_{\mathrm{acc}}$ but the truth is that we need to reach as high as possible accelerating gradient $E_{\text {acc }}$ before field emission or magnetic quench limit further increase of the accelerating gradient. So, the ideal situation would be to reach both limits simultaneously using all the possibilities to increase $E_{\text {acc }}$. If we know the maximal achievable surface peak fields $E_{\mathrm{pk}}^{*}$ and $B_{\mathrm{pk}}^{*}$, then the cavity having equal values of $E_{\mathrm{pk}} / E_{\mathrm{pk}}^{*}$ and $B_{\mathrm{pk}} / B_{\mathrm{pk}}^{*}$ will be at equal distances from either limit. Then the criterion of the shape optimization can be written as a minimum of the maximum of two values: $E_{\mathrm{pk}} / E_{\mathrm{pk}}^{*}$ and $B_{\mathrm{pk}} / B_{\mathrm{pk}}^{*}$, or, shortly, min max $\left(E_{\mathrm{pk}} / E_{\mathrm{pk}}^{*}, B_{\mathrm{pk}} / B_{\mathrm{pk}}^{*}\right)$. We can name this approach the equidistant optimization.

In the optimization, absolute values of $E_{\mathrm{pk}}$ and $B_{\mathrm{pk}}$ do not matter, only their ratio is important. Absolute values depend on normalization used in the program for the cavity calculation, e.g., the total electromagnetic energy stored in the cavity or the accelerating rate. Their ratio depends on the geometry only. Values under the sign of minmax (see above) become equal in the result because $E_{\mathrm{pk}}$ and $B_{\mathrm{pk}}$ change reversely: when one of them increases, the other decreases, and vice versa.

The same statement about ratio is true for $E_{\mathrm{pk}}^{*}$ and $B_{\mathrm{pk}}^{*}$ : optimization for $E_{\mathrm{pk}}^{*}=120 \mathrm{MV} / \mathrm{m}$ and $B_{\mathrm{pk}}^{*}=240 \mathrm{mT}$ will be the same as optimization for $E_{\mathrm{pk}}^{*}=100 \mathrm{MV} / \mathrm{m}$ and $B_{\mathrm{pk}}^{*}=200 \mathrm{mT}$. We can consider the first pair of parameters as the aggressive version for the future cavities, and the second pair as a basic version. In this case, we need to do only one optimization for both cases. Let us call this optimization "optimization 100/200."

A possible future progress in the increase of achievable fields can change this proportion, and we have this proportion changed [10]: a gradient of $59 \mathrm{MV} / \mathrm{m}$ was achieved in a single-cell cavity that corresponds to a peak surface electric field of $125 \mathrm{MV} / \mathrm{m}$ and a peak magnetic field of $206.5 \mathrm{mT}$. The gradient was limited by a hard quench. We will make another optimization with these parameters $125 / 206.5 \approx 120 / 200$ and call it "optimization 120/200." The comprehension that quench governed by $B_{\mathrm{pk}}$ is a hard limit, whereas field emission, governed by $E_{\mathrm{pk}}$, could be decreased with better cleaning made possible to achieve this record gradient.

Optimization for minimum $B_{\mathrm{pk}} / E_{\mathrm{acc}}$ when the value of $E_{\mathrm{pk}} / E_{\mathrm{acc}}$ is given, can be revised in the light of the method proposed here. For example, well optimized for a given aperture, the TESLA cavity has $E_{\mathrm{pk}} / E_{\mathrm{acc}}=2$ and $B_{\mathrm{pk}} / E_{\mathrm{acc}}=4.2 \mathrm{mT} /(\mathrm{MV} / \mathrm{m})$. If we assume that both limits, $E_{\mathrm{pk}}^{*}$ and $B_{\mathrm{pk}}^{*}$, are achieved simultaneously in this optimization, then $E_{\mathrm{pk}} / B_{\mathrm{pk}}=$ $E_{\mathrm{pk}}^{*} / B_{\mathrm{pk}}^{*}=2 / 4.2(\mathrm{MV} / \mathrm{m}) / \mathrm{mT}=100 / 210(\mathrm{MV} / \mathrm{m}) / \mathrm{mT}$. This means that this cavity can be treated as a cavity 
TABLE III. Parameters of optimized cells with limiting surface fields: (1) $E_{\mathrm{pk}}^{*}=100 \mathrm{MV} / \mathrm{m}$ and $B_{\mathrm{pk}}^{*}=200 \mathrm{mT}$, and (2) $E_{\mathrm{pk}}^{*}=120 \mathrm{MV} / \mathrm{m}$ and $B_{\mathrm{pk}}^{*}=200 \mathrm{mT} ; L-A=5 \mathrm{~mm}$, aperture radius $\mathrm{R}_{a}=25 \mathrm{~mm}$. $E_{\mathrm{acc}}^{*}$ is the accelerating rate when the limiting surface fields are achieved.

\begin{tabular}{|c|c|c|c|c|c|c|}
\hline Optimization & $100 / 200$ & $120 / 200$ & $120 / 200$ & $120 / 200$ & $120 / 200$ & $120 / 200$ \\
\hline Phase advance $\theta$, deg & 105 & 90 & 95 & 100 & 105 & 110 \\
\hline$A, \mathrm{~mm}$ & 28.631 & 23.826 & 25.428 & 27.029 & 28.631 & 30.232 \\
\hline$B, \mathrm{~mm}$ & 97.44 & 36.4 & 38.1 & 39.9 & 40.91 & 42.1 \\
\hline$a, \mathrm{~mm}$ & 6.084 & 4.512 & 4.840 & 5.171 & 5.494 & 5.817 \\
\hline$b, \mathrm{~mm}$ & 11.098 & 7.52 & 8.136 & 8.772 & 9.379 & 9.986 \\
\hline$E_{\mathrm{pk}} / E_{\mathrm{acc}}$ & 1.655 & 1.727 & 1.730 & 1.734 & 1.739 & 1.745 \\
\hline$B_{\mathrm{pk}} / E_{\mathrm{acc}}, \mathrm{mT} /(\mathrm{MV} / \mathrm{m})$ & 3.309 & 2.878 & 2.883 & 2.890 & 2.898 & 2.909 \\
\hline$R_{\mathrm{sh}} / Q, \mathrm{Ohm} / \mathrm{m}$ & 1789 & 2127 & 2096 & 2063 & 2029 & 1992 \\
\hline$\alpha$, degrees & 94.73 & 90.91 & 90.33 & 89.61 & 88.77 & 87.71 \\
\hline$R_{\mathrm{eq}}, \mathrm{mm}$ & 106.156 & 98.950 & 98.991 & 99.068 & 99.016 & 99.011 \\
\hline$v_{\mathrm{gr}} / c$ & 0.01365 & 0.01831 & 0.01776 & 0.01710 & 0.01635 & 0.01551 \\
\hline$E_{\mathrm{acc}}^{*}, \mathrm{MV} / \mathrm{m}$ & 60.4 & 69.5 & 69.4 & 69.2 & 69.0 & 68.8 \\
\hline$E_{\mathrm{acc}}^{*} \times 2 L, \mathrm{MV}$ & 4.06 & 4.00 & 4.22 & 4.43 & 4.64 & 4.85 \\
\hline
\end{tabular}

optimized for $E_{\mathrm{pk}}^{*}=100 \mathrm{MV} / \mathrm{m}$ and $B_{\mathrm{pk}}^{*}=210 \mathrm{mT}$, or, proportionally, for example, for $E_{\mathrm{pk}}^{*}=80 \mathrm{MV} / \mathrm{m}$ and $B_{\mathrm{pk}}^{*}=168 \mathrm{mT}$. (Nevertheless, the method of equidistant optimization can be systematically applied for optimization of SW cavities).

The difference between these two methods is in the fact that we do not know a priori what value of $B_{\mathrm{pk}} / E_{\mathrm{acc}}$ we will have for a given value of $E_{\mathrm{pk}} / E_{\mathrm{acc}}$ in the old method, but in the new method, we can choose the ratio between the extremal fields based on experiment, and then perform the optimization.

It was a lucky case that ratio $E_{\mathrm{pk}} / E_{\mathrm{acc}}=2$ was chosen for optimization of the TESLA cavity. Even a better choice $E_{\mathrm{pk}} / E_{\mathrm{acc}}=2.4$ was used for the reentrant cavity because it appeared that this cavity could reach a record accelerating gradient [10]. Optimization for minimum $B_{\mathrm{pk}} / E_{\mathrm{acc}}$ by this given value of $E_{\mathrm{pk}} / E_{\mathrm{acc}}=2$ resulted in a ratio " $120 / 200$ " in our short designation for the $\left(E_{\mathrm{pk}} / E_{\mathrm{pk}}^{*}, B_{\mathrm{pk}} / B_{\mathrm{pk}}^{*}\right)$. The obtained values $125 \mathrm{MV} / \mathrm{m}$ and $206.5 \mathrm{mT}$ really look like maximal fields for existing cavities. However, if we knew that these fields are maximum achievable, we will find the optimal shape from the first try.
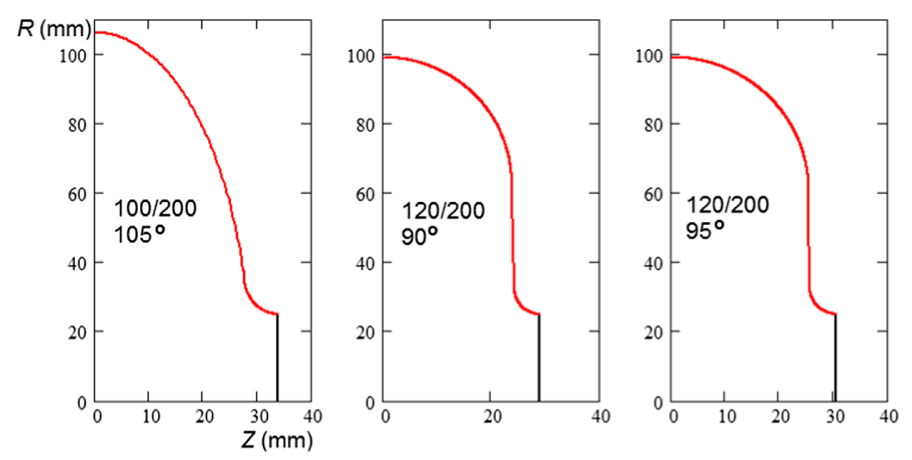

In the case of a TW, when we hope for a higher than $65 \mathrm{MV} / \mathrm{m}$ acceleration rate, we cannot use the value $E_{\mathrm{pk}} / E_{\mathrm{acc}}=2.4$ or 2 . Values of $E_{\mathrm{pk}} / E_{\mathrm{acc}}$ and $B_{\mathrm{pk}} / E_{\mathrm{acc}}$ will be obtained as a result of optimization when limiting fields are given. As can be seen below, $E_{\mathrm{pk}} / E_{\mathrm{acc}}$ appeared to be substantially less than 2 .

The procedure of optimization for minmax $\left(E_{\mathrm{pk}} / E_{\mathrm{pk}}^{*}\right.$, $B_{\mathrm{pk}} / B_{\mathrm{pk}}^{*}$ ) consisted in a systematical change of the elliptical half axes $A, B, a$, and $b$ [Fig. 2(b)] decreasing maximal value in parentheses, as a result both ratios become equal.

We hope that the novelty of this optimization method warrants a more complete study in comparison with conventional methods for both SW and TW cavities.

\section{FIRST OPTIMIZATIONS 100/200 AND 120/200 FOR A TRAVELING WAVE. APERTURE $2 R_{a}=50 \mathrm{~mm}$}

Let us use the approach described above for optimization of a cell for a periodic structure with an aperture $2 R_{a}=50 \mathrm{~mm}$. We assume the limiting surface fields as (1) $E_{\mathrm{pk}}^{*}=100 \mathrm{MV} / \mathrm{m}$ and $B_{\mathrm{pk}}^{*}=200 \mathrm{mT}$, and (2) $E_{\mathrm{pk}}^{*}=120 \mathrm{MV} / \mathrm{m}$ and $B_{\mathrm{pk}}^{*}=200 \mathrm{mT}$. The phase
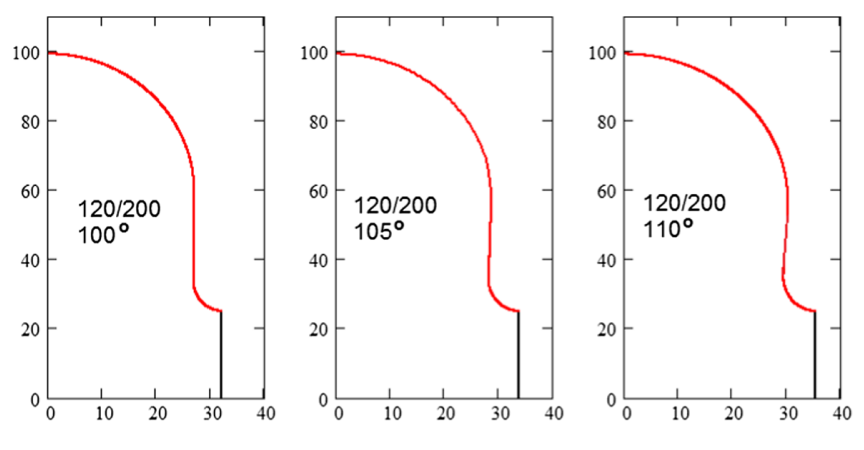

FIG. 5. Cells with parameters presented in Table III. 
TABLE IV. Parameters of an optimized cell with the limiting surface fields $E_{\mathrm{pk}}^{*}=120 \mathrm{MV} / \mathrm{m} \quad$ and $B_{\mathrm{pk}}^{*}=200 \mathrm{mT}$; $L-A=5 \mathrm{~mm}$, aperture radius $\mathrm{R}_{a}=20 \mathrm{~mm}$. $E_{\mathrm{acc}}^{*}$ is the accelerating rate when the limiting surface fields are achieved.

\begin{tabular}{lc}
\hline \hline Optimization & $120 / 200$ \\
\hline Phase advance $\theta, \mathrm{deg}$ & 90 \\
$A, \mathrm{~mm}$ & 23.826 \\
$B, \mathrm{~mm}$ & 35 \\
$a, \mathrm{~mm}$ & 3.874 \\
$b, \mathrm{~mm}$ & 6.777 \\
$E_{\mathrm{pk}} / E_{\mathrm{acc}}$ & 1.639 \\
$B_{\mathrm{pk}} / E_{\mathrm{acc}}, \mathrm{mT} /(\mathrm{MV} / \mathrm{m})$ & 2.732 \\
$R_{\mathrm{eq}} / Q, \mathrm{Ohm} / \mathrm{m}$ & 2367 \\
$\alpha, \mathrm{degrees}$ & 91.74 \\
$R_{\mathrm{eq}}, \mathrm{mm}$ & 97.990 \\
$v_{\mathrm{gr}} / c$ & 0.009315 \\
$E_{\mathrm{acc}}^{*}, \mathrm{MV} / \mathrm{m}$ & 73.2 \\
$E_{\mathrm{acc}}^{*} \times 2 L, \mathrm{MV}$ & 4.22 \\
\hline
\end{tabular}

advance is $\theta=105^{\circ}$ for the case (1) and varies from $90^{\circ}$ to $110^{\circ}$ for the case (2).

We will omit here details of the optimization, and present results only, see Table III and Fig. 5. A value of $E_{\mathrm{pk}} / E_{\mathrm{acc}}$ is low, as compared with standing wave cavities; possibly, this is an effect of transition to TW. The values of $R_{\mathrm{sh}} / Q$, responsible for the dynamic heat load, are about 2 times higher in all further optimizations (Tables IV and V) of the TW structures than in the SW. Comparison with the TESLA cavity is presented in Table VI.

To make this comparison between SW and TW structures fairer, we added in Table VI an optimization of the TW structure with the same aperture as in the TESLA structure. It should be added, however, that a SW structure with aperture radius of $25 \mathrm{~mm}$, by contrast with a TW structure,

TABLE V. Parameters of optimized cells with limiting surface fields (1) $E_{\mathrm{pk}}^{*}=115 \mathrm{MV} / \mathrm{m}$ and $B_{\mathrm{pk}}^{*}=200 \mathrm{mT}$, (2) $E_{\mathrm{pk}}^{*}=$ $120 \mathrm{MV} / \mathrm{m}$ and $B_{\mathrm{pk}}^{*}=200 \mathrm{mT}$, and (3) $E_{\mathrm{pk}}^{*}=125 \mathrm{MV} / \mathrm{m}$ and $B_{\mathrm{pk}}^{*}=200 \mathrm{mT} ; \quad a=L-A=5 \mathrm{~mm}$, aperture radius $R_{a}=25 \mathrm{~mm} . E_{\mathrm{acc}}^{*}$ is the accelerating rate when the limiting surface fields are achieved.

\begin{tabular}{lccc}
\hline \hline Optimization & $115 / 200$ & $120 / 200$ & $125 / 200$ \\
\hline $\begin{array}{l}\text { Phase advance } \theta, \mathrm{deg} \\
\quad \text { advance } q, \mathrm{deg}\end{array}$ & 100 & 100 & 100 \\
$B, \mathrm{~mm}$ & 68.1 & 51.5 & 38.8 \\
$b, \mathrm{~mm}$ & 8.222 & 8.258 & 7.422 \\
$E_{\mathrm{pk}} / E_{\mathrm{acc}}$ & 1.744 & 1.752 & 1.801 \\
$B_{\mathrm{pk}} / E_{\mathrm{acc}}, \mathrm{mT} /(\mathrm{MV} / \mathrm{m})$ & 3.035 & 2.919 & 2.882 \\
$R_{\mathrm{sh}} / Q, \mathrm{Ohm} / \mathrm{m}$ & 1957 & 2029 & 2072 \\
$R_{\mathrm{eq}}, \mathrm{mm}$ & 103.609 & 101.192 & 98.896 \\
$v_{\mathrm{gr}} / c$ & 0.01626 & 0.01695 & 0.01705 \\
$E_{\mathrm{acc}}^{*}, \mathrm{MV} / \mathrm{m}$ & 65.9 & 68.5 & 69.4 \\
$E_{\mathrm{acc}}^{*} \times 2 L, \mathrm{MV}$ & 4.22 & 4.39 & 4.45 \\
\hline \hline
\end{tabular}

would have a lot of problems with trapped modes and weak cell-to-cell coupling.

All most important parameters $-E_{\mathrm{pk}} / E_{\mathrm{acc}}, B_{\mathrm{pk}} / E_{\mathrm{acc}}$, $R_{\mathrm{sh}} / Q, E_{\mathrm{acc}}^{*}$-are improving when the phase advance per cell $\theta$ decreases. However, acceleration per cell, $E_{\text {acc }}^{*} \times 2 L$, reduces, and the shorter the cell the less acceleration it provides ( $2 L$ is the cell length). Because of high cost of superconducting cell fabrication, the number of cells should be minimized. It can also be shown that requirements to the accuracy of dimensions are more stringent when the dimensions defining the cell's shape decrease. So, the trade-off for the phase advance per cell should be defined. A possible solution is a cell with the wall slope angle $\alpha=90^{\circ}$ that corresponds to $\theta$ between $95^{\circ}$ and $100^{\circ}$. Cells with $\alpha=90^{\circ}$ seem simpler for manufacturing and chemical treatment compared to reentrant cells.

\section{OPTIMIZATIONS 120/200 FOR A TRAVELING WAVE. APERTURE $2 R_{a}=40 \mathrm{~mm}$}

To investigate benefits of a smaller aperture, data for a cavity with $R_{a}=20 \mathrm{~mm}$ are presented in Table IV. Phase advance of $90^{\circ}$ is chosen; as can be seen from Table III, dependence of $E_{\mathrm{acc}}^{*}$ on this value is not strong.

VIII. COMPARISON OF OPTIMIZATIONS 115/200, 120/200, AND 125/200. APERTURE $2 R_{a}=50 \mathrm{~mm}$, WALL SLOPE ANGLE $\alpha=90^{\circ}$, PHASE ADVANCE $\theta=100^{\circ}, a=5 \mathrm{~mm}$

To understand benefits of further improvement of the surface for decreasing the dark currents, optimization was done for a simplified geometry with $A+a=L$, where $a=5 \mathrm{~mm}$, i.e., for the wall slope angle $\alpha=90^{\circ}$. The phase advance was taken $\theta=100^{\circ}$ because in previous optimization this phase advance led to $\alpha$ close to $90^{\circ}$. Results are presented in Table V.

Figure 6 illustrates data of the Table $\mathrm{V}$ for the same parameters $\theta, a$, and $R_{a}$. Values of $E_{\text {acc }}^{*}$ are recalculated to 65,70 , and $75 \mathrm{MV} / \mathrm{m}$ using proportionality between $E_{\mathrm{pk}}^{*}$, $B_{\mathrm{pk}}^{*}$, and $E_{\mathrm{acc}}^{*}$. As it was shown above, the optimized shape is the same if values of $E_{\mathrm{pk}}^{*}$ and $B_{\mathrm{pk}}^{*}$ are proportionally changed. However, the achievable accelerating rate will also change proportionally. So, for example, the optimized shape $125 / 200$ is the same as the shape for 126.1/201.7, but the maximal accelerating rate in this case will be $70 \mathrm{MV} / \mathrm{m}$ instead of 69.4 .

Figure 6 shows that for the parameters given above it is impossible to reach $E_{\mathrm{acc}}^{*}=70 \mathrm{MV} / \mathrm{m}$ with $B_{\mathrm{pk}}^{*}=200 \mathrm{mT}$, however high the $E_{\mathrm{pk}}^{*}$ may be. An analogous statement is true for $E_{\mathrm{pk}}^{*}=120 \mathrm{MV} / \mathrm{m}$ when the $B_{\mathrm{pk}}^{*}$ increases.

\section{MULTIPACTOR CONSIDERATION}

According to [23], existence of a multipactor in a cavity is defined by the geometrical parameter $p$, Fig. 7; 
TABLE VI. Parameters of some TW cells from Table III before and after transformation removing multipactor conditions. Two right columns-parameters of a TW cavity with the same aperture as the TESLA cavity for comparison.

\begin{tabular}{|c|c|c|c|c|c|}
\hline Optimization & $120 / 200$ & $120 / 200$ & $120 / 200$ & $120 / 200$ & TESLA $(100 / 210)$ \\
\hline \multirow[t]{2}{*}{ Phase advance $\theta$, degrees } & 90 & 95 & 100 & 90 & $180(\mathrm{SW})$ \\
\hline & Before/after & Before/after & Before/after & Before/after & \\
\hline$A, \mathrm{~mm}$ & 23.826 & 25.428 & 27.029 & 23.826 & 42 \\
\hline$B, \mathrm{~mm}$ & $36.4 / 24$ & $38.1 / 28$ & $39.9 / 30$ & $38 / 26$ & 42 \\
\hline$a, \mathrm{~mm}$ & 4.512 & 4.840 & 5.171 & 5.40 & 12 \\
\hline$b, \mathrm{~mm}$ & 7.52 & 8.136 & 8.772 & 7.89 & 19 \\
\hline$E_{\mathrm{pk}} / E_{\mathrm{acc}}$ & $1.727 / 1.728$ & $1.730 / 1.730$ & $1.734 / 1.734$ & $1.923 / 1.923$ & 1.99 \\
\hline$\delta\left[E_{\mathrm{pk}} / E_{\mathrm{acc}}\right], \%$ & 0.081 & 0.010 & 0.013 & 0.000 & \\
\hline$B_{\mathrm{pk}} / E_{\mathrm{acc}}, \mathrm{mT} /(\mathrm{MV} / \mathrm{m})$ & $2.878 / 2.897$ & $2.883 / 2.904$ & $2.890 / 2.913$ & $3.206 / 3.230$ & 4.16 \\
\hline$\delta\left[B_{\mathrm{pk}} / E_{\mathrm{acc}}\right], \%$ & 0.651 & 0.734 & 0.809 & 0.736 & \\
\hline$R_{\mathrm{sh}} / Q, \mathrm{Ohm} / \mathrm{m}$ & $2127 / 2151$ & $2096 / 2115$ & $2063 / 2081$ & $1675 / 1690$ & 993 \\
\hline$R_{\mathrm{eq}}, \mathrm{mm}$ & $98.950 / 96.458$ & $98.991 / 97.002$ & $98.569 / 97.144$ & $101.838 / 99.624$ & 103.35 \\
\hline Aperture radius $R_{a}, \mathrm{~mm}$ & 25 & 25 & 25 & 35 & 35 \\
\hline$p$ & $0.302 / 0.270$ & $0.301 / 0.278$ & $0.303 / 0.279$ & $0.305 / 0.279$ & 0.286 \\
\hline
\end{tabular}

experimental data presented in this book show that at $p=$ 0.3 and higher there is a strong multipactor. The TESLA shape cavity has $p=0.286$ and has a weak multipactor activity. The Cornell ERL cavity has $p=0.276$, and a multipactor in it was not observed. With some degree of caution, one can say that $p=0.28$ is a safe limit for a multipactor. The case in point is the elliptical niobium superconducting cavity with a standard treatment of the surface, other materials can give different limits of $p$. We define $p$ for a two-point multipactor to which the elliptical cavities are susceptible foremost. A one-point multipactor

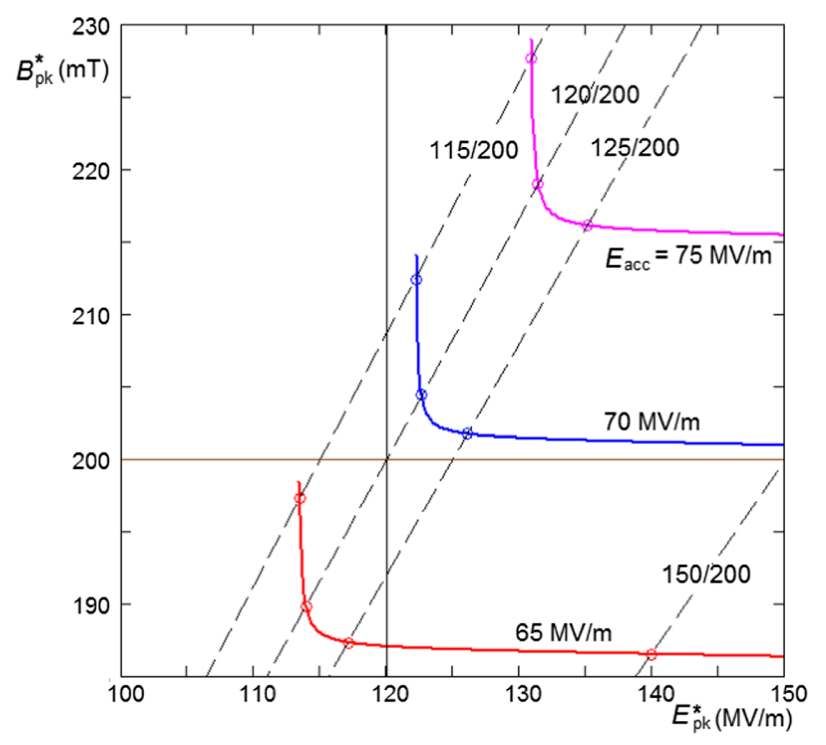

FIG. 6. Maximal achievable accelerating rates $E_{\text {acc }}^{*}$ for different limiting surface fields $E_{\mathrm{pk}}^{*}$ and $B_{\mathrm{pk}}^{*}$. The dashed lines show different optimization options. The optimized cavity shape on each dashed line is the same. Optimization is done for conditions presented in Table V. occurs if there is a long flat region in the cavity equator area that is not our case.

In the TW regime, in distinction to $\mathrm{SW}$, the maximum of $E$ or $H$ field is reached at different times for different points. For this reason, the fields can be presented in the form, e.g., $H(r, z, t)=U(r, z, t)+i V(r, z, t)$, where functions $U$ and $V$ reach their maxima with a shift in phase of $90^{\circ}$. Fortunately, the function $V$ is small compared to $U$ in the equator area, $L=0$ in Fig. 8, and all the theory appears quite applicable to the TW regime as it is for the SW regime.

If we calculate $p$ for cavities presented above, we will see that this parameter is higher than 0.28 and a multipactor in some cases is inevitable. A solution of this problem can be a change of the cell shape that does not compromise the optimized parameters too much (say, not more than $1 \%$ for $E_{\mathrm{pk}} / E_{\mathrm{acc}}$ and $\left.B_{\mathrm{pk}} / E_{\mathrm{acc}}\right)$ but decreases $p$ to a safe value. A possible way to decrease $p$ is in an increase of the curvature radius at the cavity equator [formula (8.16) in [23]]. A change in the iris half-axes $a$ and $b$ has a small influence on the value of $p$ and leads to a fast change in the value of $E_{\mathrm{pk}}$, the value of half-axis $A$ is chosen as big as possible and cannot be increased (technological limitations) to decrease

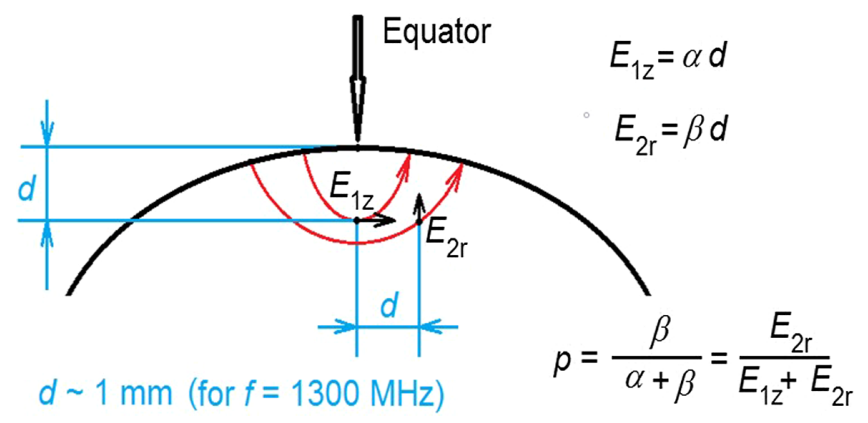

FIG. 7. Definition of the parameter $p$, from [22]. 


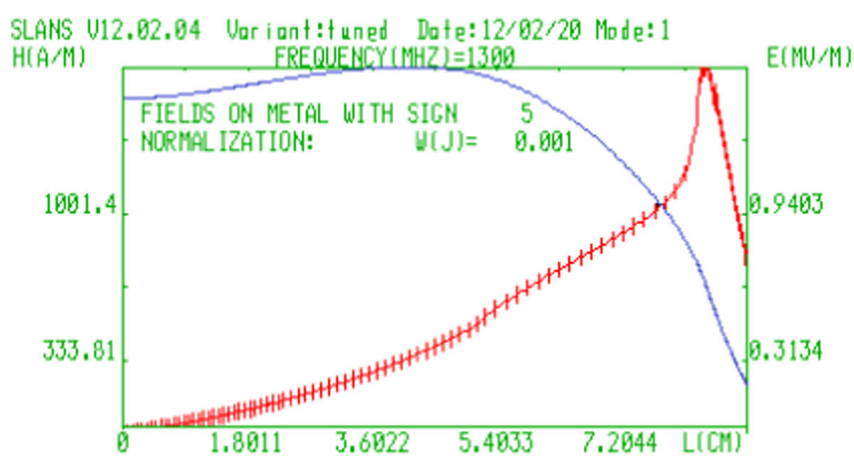

SLANS U12.02.04 Uar iant:tuned Date: 12/02/20 Mode: 1

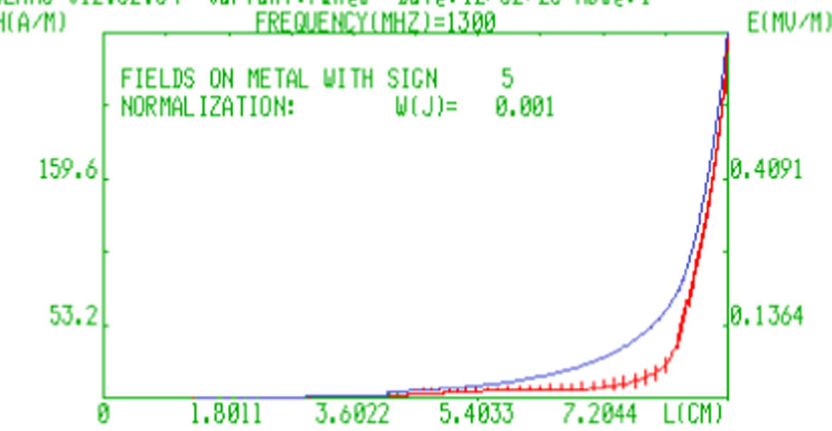

FIG. 8. $U$ (left picture) and $V$ (right) components of the electric (red) and magnetic (blue) surface fields along the profile line for the geometry from the second column of Table V.

$B_{\mathrm{pk}}$. So, we will decrease the length of the half-axis $B$ that is perpendicular to the cavity axis, and so increase the curvature radius at the equator $R_{c}=A^{2} / B$.

As an example, let us consider the cavity with $\theta=100^{\circ}$, $R_{a}=25 \mathrm{~mm}$, optimized for " $120 / 200$," see Table III. Its original $p=0.303$. When $B$ is decreased from 39.9 to $30 \mathrm{~mm}, E_{\mathrm{pk}} / E_{\mathrm{acc}}=1.734$ will remain practically the same with a slight increase of $0.013 \%, B_{\mathrm{pk}} / E_{\mathrm{acc}}=2.890$ will increase to $2.913 \mathrm{mT} /(\mathrm{MV} / \mathrm{m})$, i.e., by $0.81 \%$. To keep the frequency, $f=1300 \mathrm{MHz}$, the equatorial radius will change from 99.068 to $97.144 \mathrm{~mm}$. The new value of $p$ will be 0.279 .

The relative change of $E_{\mathrm{pk}} / E_{\mathrm{acc}}$ and $B_{\mathrm{pk}} / E_{\mathrm{acc}}$ is designated in Table VI as $\delta\left[E_{\mathrm{pk}} / E_{\mathrm{acc}}\right]$ and $\delta\left[B_{\mathrm{pk}} / E_{\mathrm{acc}}\right]$, in percent. The accelerating rate when one of the limiting surface fields is achieved, $E_{\mathrm{acc}}^{*}$, will also decrease relative to the original value, but, again, not more than $1 \%$.

Such a transformation was done for several cell shapes from Table III. Results are presented in Table VI.

So, here the method of anti-multipactoral transformation is presented, and hopefully any shape of a cell chosen in the future can be transformed into a multipactor-free shape without big losses in the acceleration rate.

\section{GROUP VELOCITY AND CAVITY LENGTH LIMITATIONS}

In this section we estimate the maximum length for TW structures, assuming that the same maximum level of field errors shall be reached, which is present in SW cavities. Potential gains for long TW structures are also assessed.

Coupling coefficient $k$ is related to the group velocity as follows:

$$
k=2 \beta /(\theta \sin \theta),
$$

where $\theta$ is the phase advance per cell, $\beta=v_{g r} / c$ is the group velocity normalized to speed of light. All of the examples of cavities in Table III have $\beta>0.01$. For a group velocity 0.01 and $\theta=105^{\circ}$ we have $k=1.13 \times 10^{-2}$. We know that a SW $\pi$-mode structure can be tuned, it has $k_{\mathrm{SW}}=1.8 \times 10^{-2}$. To have the same error in the field, we must have the number of cells in a TW structure equal to

$$
N_{\mathrm{TW}}=2\left(k_{\mathrm{TW}} / k_{\mathrm{SW}}\right) \cdot N_{\mathrm{SW}}^{2} \text {. }
$$

The length of this structure will be $L_{s}=N_{\mathrm{TW}} \cdot L_{c}$, where $L_{c}$ is length of a cell, $L_{c}=\lambda \theta /(2 \pi)$. Uniting all the above expressions, one can write

$$
L_{s}=\frac{2 N_{\mathrm{SW}}^{2}}{\pi k_{\mathrm{SW}}} \cdot \frac{\beta \lambda}{\sin \theta} .
$$

Even for $\sin \theta=1$ the length of the structure will be 6.6 meters; it is much longer than can be permitted by technological limitations. Even a small aperture structure (Table IV) can be long enough having $\beta=0.009$, close to 0.01 . If a TW structure of length $2.4 \mathrm{~m}$ can be realized, and if we compare the active structure length of a TW solution with an ILC-type nine-cell SW solution (assuming the same accelerating gradient), then the resulting real-estate gradient would be $20 \%$ higher (Fig. 9). This assumes a ninecell structure length of $1 \mathrm{~m}$ and $40 \mathrm{~cm}$ distance between the cavities.

For a TW cavity it means a real-estate gradient $84 \mathrm{MeV} / \mathrm{m}$ instead of $70 \mathrm{MeV} / \mathrm{m}$.

\section{INFLUENCE OF FABRICATION ERRORS}

Dimensions of the cells' elements in the Tables above are often given with an accuracy of micrometers. These are theoretical values which barely can be reached. We need to define deviations of these dimensions that do not
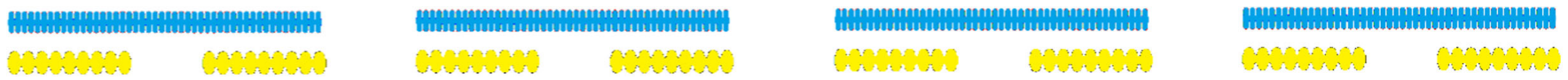

FIG. 9. Removing nonaccelerating intervals between cavities: comparison with the TESLA cryomodule comprising eight cavities. 

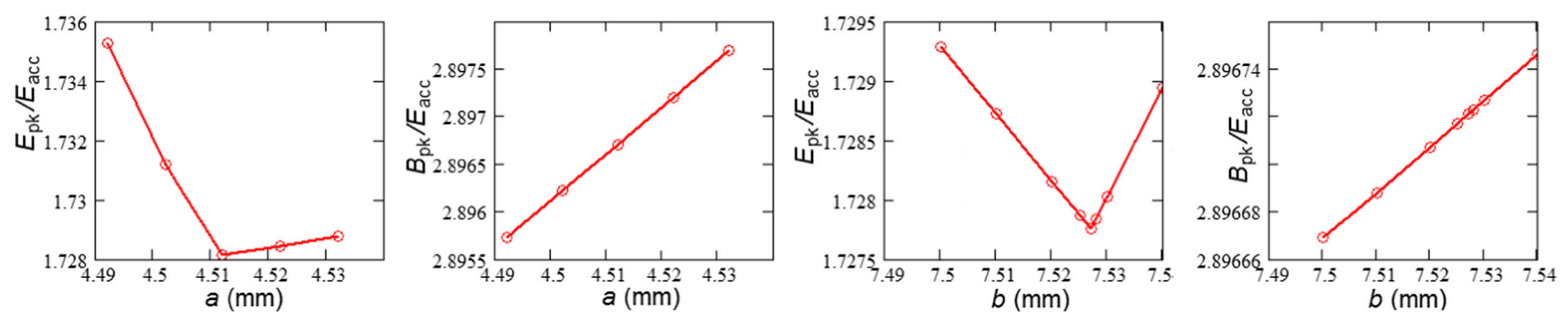

FIG. 10. Dependencies of $E_{\mathrm{pk}} / E_{\mathrm{acc}}$ and $B_{\mathrm{pk}} / E_{\mathrm{acc}}$ on variations of lengths $a$ and $b$ for a cavity cell with $\theta=90^{\circ}$ and $B=24 \mathrm{~mm}$ from Table VI.

compromise important figures of merit. The value of $B$, the half axis of the big ellipse, was already discussed: we changed it within several millimeters and had $E_{\mathrm{pk}} / E_{\mathrm{acc}}$ and $B_{\mathrm{pk}} / E_{\mathrm{acc}}$ changed within $1 \%$. Only slightly more sensitive are these parameters to deviation of $A$, another half axis of the big ellipse. Half axes of the small ellipse, $a$ and $b$, are much shorter than in the case of a $\pi$-mode SW structure (TESLA, e.g., has $a=12 \mathrm{~mm}$ and $b=19 \mathrm{~mm}$ ). The sensitivity of the peak fields to the values of $a$ and $b$ is shown in Fig. 10. Contrary to the analytic functions, the derivative of the $E_{\mathrm{pk}} / E_{\mathrm{acc}}$ relative to $a$ or $b$ at its minimum is not zero but changes its value and sign at the point of optimum.

For the case presented in Fig. 10, the growth of $E_{\mathrm{pk}} / E_{\mathrm{acc}}$ is $1 \%$ when $a$ increases by $0.580 \mathrm{~mm}$, and this growth is also $1 \%$ when $a$ decreases by $0.058 \mathrm{~mm}$, so the sensitivity differs 10 times. The value of $B_{\mathrm{pk}} / E_{\mathrm{acc}}$ depends linearly on $a$ and changes by $\pm 1 \%$ when $a$ changes by $\pm 0.590 \mathrm{~mm}$.

The growth of $E_{\mathrm{pk}} / E_{\mathrm{acc}}$ is $1 \%$ for an increase of $b$ by $0.190 \mathrm{~mm}$ or a decrease by $0.305 \mathrm{~mm}$. Sensitivity of $B_{\mathrm{pk}} / E_{\mathrm{acc}}$ to the change of $b$ is about $0.06 \%$ per $1 \mathrm{~mm}$.

Sensitivities of other geometries presented in Table VI to fabrication errors are close to the values displayed here.

\section{LOSSES AND FIELDS IN THE FEEDBACK WAVEGUIDE}

It is supposed that a traveling wave structure is used in a resonant ring configuration [13-16]. Hence, a portion of the circulating power will be absorbed in the feedback waveguide and decrease the efficiency of the structure. In this section we estimate this effect.

The $Q$ factor of a structure is

$$
Q=\frac{\omega_{0} W}{P_{\text {loss }}},
$$

where $\omega_{0}$ is the operating frequency, $W$ is the stored energy, and $P_{\text {loss }}$ is power loss in the structure, so

$$
W=\frac{P_{\text {loss }} Q}{\omega_{0}} .
$$

An energy per unit length is $W / L_{s}$, where $L_{s}$ is the structure length, and a flow of power through any cross section of the structure is

$$
P=\frac{W}{L_{s}} \cdot v_{\mathrm{gr}}=\frac{P_{\mathrm{loss}} Q}{L_{s} \omega_{0}} \cdot v_{\mathrm{gr}},
$$

where $v_{\mathrm{gr}}$ is the group velocity. Here we assumed that the power density is the same in any cross section, or power loss is much less than power circulating in the ring: $P_{\text {loss }} \ll P$.

Power loss can be expressed as

$$
P_{\text {loss }}=\frac{V^{2}}{R},
$$

$V$ is the cavity voltage; $R$ is the cavity shunt impedance. So, the power circulating in the ring is

$$
P=\frac{V^{2}}{R} \cdot \frac{Q}{L_{s} \omega_{0}} \cdot v_{\mathrm{gr}}=\frac{V^{2}}{R_{\mathrm{sh}} / Q} \cdot \frac{v_{\mathrm{gr}}}{L_{s}^{2} \omega_{0}} .
$$

Here $R_{\mathrm{sh}} / Q$ is in $\mathrm{Ohm} / \mathrm{m}$, whereas $R$ is measured in Ohms. Therefore, $L_{s}$ appears squared.

Using $L_{s}=1 \mathrm{~m}, V=70 \mathrm{MV}, R_{\mathrm{sh}} / Q=2000 \mathrm{Ohm} / \mathrm{m}$, $f=\omega_{0} / 2 \pi=1.3 \times 10^{9} \mathrm{~Hz}$, and $v_{\mathrm{gr}}=0.01 c$, we calculate the power circulating in the ring $P=900 \mathrm{MW}$. Power loss in the whole structure is

$$
P_{\text {loss }}=\frac{V^{2}}{\left(R_{\mathrm{sh}} / Q\right) Q L_{s}}=240 \mathrm{~W} .
$$

$Q$ is taken as $10^{10}$.

The attenuation coefficient of a rectangular waveguide for the $\mathrm{H}_{10}$ wave is

$$
\eta=\frac{2 k_{r} R_{s}}{b Z_{0}}\left[\frac{1}{\sqrt{K}}\left(\frac{1}{2}+\frac{h}{d} \frac{f_{\mathrm{cr}}^{2}}{f^{2}}\right)\right]
$$

where $k_{r}$ is surface roughness coefficient (here, for simplicity $\left.k_{r}=1\right), R_{s}$ is the surface resistance, $Z_{0}$ is the impedance of free space, $K=1-f_{\mathrm{cr}}^{2} / f^{2}, f_{\mathrm{cr}}=c /(2 d)$ is the critical frequency of the waveguide, and $d$ and $h$ are the transverse dimensions (width and height) of the waveguide.

Taking dimensions of a standard WR-650 waveguide $d \times \mathrm{h}=165.1 \mathrm{~mm} \times 85.55 \mathrm{~mm}$ and surface resistance of the superconducting niobium $R_{s}=27 \mathrm{nOhm}$ (this value 
corresponds to $Q=10^{10}$ for the TESLA cavity) we have $\eta=1.8 \times 10^{-9} 1 / \mathrm{m}$. Attenuation of power follows the law: $P=P_{0} \cdot \exp (-2 \eta L)$. For the given values (waveguide length $\left.L=1 \mathrm{~m}, P_{0}=900 \mathrm{MW}\right) \Delta P=P_{0}-P=$ $P_{0}[1-\exp (-2 \eta L)]=3.6 \times 10^{-9} P_{0}=3.2 \mathrm{~W}$, so losses in the waveguide are about $1.3 \%$ of the losses in the structure.

Electric and magnetic fields in the waveguide are significantly smaller than in the cavity because group velocity in the waveguide is much higher than in the cavity. All the diagnostic ports in the WG have special elliptical roundings of the opening to minimize field enhancement. For the TW design considered in this paper the electric field in the regular part of the WG does not exceed $10 \%$ of the maximal field in the periodic structure.

Quench is a considerably slow process compared to breakdown, it takes about $1 \mathrm{~ms}$ and does not damage the cavity. The quench dynamics is determined by not the circulating power, but by stored energy. The stored energy in a TW cavity is about the same as in a SW cavity of about the same length. In contrast to breakdown spot, a quench spot size is typically big, about $8-10 \mathrm{~cm}$ for the ILC-type cavity at $\sim 30 \mathrm{MV} / \mathrm{m}$, see [24].

\section{CONCLUSION}

We presented results of modeling traveling wave structures aimed to keep both $B_{\mathrm{pk}}$ and $E_{\mathrm{pk}}$ values below limiting levels. A method of equidistant optimization is proposed that considers equally distant values of fields from experimentally known limiting electric and magnetic surface fields. It is shown that a TW structure can have the accelerating gradient above $70 \mathrm{MV} / \mathrm{m}$ with the same critical magnetic field that the contemporary standing wave structures. The other demonstrated benefit of TW structures is that their $R_{\mathrm{sh}} / Q$ is about 2 times higher than for the TESLA structure, which is equivalent to a factor of 2 higher $Q$ for reducing dynamic heat load, and the rf power demand. A multipactor suppression method is proposed: by sacrificing less than $1 \%$ of the accelerating rate one can make the TW cavity multipactor-free. A group velocity for all simulated structures was calculated. The results show that cell-to-cell coupling is high enough to permit a very long cavity, so that the length will be limited only by fabrication considerations. An estimation of tolerances for fabrication of the cavity cells is done. A design solution with the iris disk is proposed that can ease welding of the cells together and improve quality of the iris surface.

Many significant challenges remain on the path toward development of practical TW niobium structures. But the time scale for accomplishing this is several decades before the ILC $3 \mathrm{TeV}$ upgrade is ready to launch. Below we list some of the challenges. There is an increased complexity due to doubling the number of cells (e.g., more welds). The cavity fabrication and surface processing fixtures and procedures must be modified and qualified. High circulating power in the feedback waveguide must be demonstrated. Damping of HOMs (higher order modes) must be studied. Preliminary results show that the first ten monopole modes up to $7 \mathrm{GHz}$ show no trapping. At 3 times lower bunch charge for the ILC $3 \mathrm{TeV}$, HOM generation is much reduced over the ILC 0.5 and $1 \mathrm{TeV}$ cases. The smaller aperture proposed in this work means higher transverse wakes, but again the 3 times lower bunch charge helps. All the challenges are magnified if structures longer than $1 \mathrm{~m}$ are to be developed to further increase the gradients.

A shorter version of this work was presented at SRF 2021 as a PowerPoint and a paper. More details of this work can be found in [25].

\section{ACKNOWLEDGMENTS}

The authors want to express great gratitude to Sergey Belomestnykh for his support and help.

[1] P. Bambade et al., Reports No. DESY 19-037, No. FERMILAB-FN-1067-PPD, No. IFIC/19-10, No. IRFU19-10, No. JLAB-PHY-19-2854, No. KEK Preprint 201892, No. LAL/RT 19-001, No. PNNL-SA-142168, and No. SLAC-PUB-17412; arXiv:1903.01629.

[2] L. Evans and S. Michizono, Reports No. KEK 2017-3, No. DESY 17-180, and No. CERN-ACC-2017-0097.

[3] T. Behnke et al., The International Linear Collider technical design report-Volume 1, executive summary, arXiv:1306.6327.

[4] A. Yamamoto, Report No. ESPP-Symp-2019-ay-190502.

[5] A. Grasselino et al., Accelerating fields up to $49 \mathrm{MV} / \mathrm{m}$ in TESLA-shape superconducting rf niobium cavities via $75 \mathrm{C}$ vacuum bake, arXiv:1806.09824.

[6] F. Furuta et al. Fermilab EP facility improvements, in Proceedings of the 19th International Conferebce on RF Superconductivity, SRF2019, Dresden, Germany (JACoW Publishing, Geneva, 2019), TUP022, p. 453, 10.18429/ JACoW-SRF2019-TUP022.

[7] W. Singer et al., Development of large grain cavities, Phys. Rev. ST Accel. Beams 16, 012003 (2013).

[8] F. Furuta et al., Experimental comparison at KEK of high gradient performance of different single cell superconducting cavity designs, in Proceedings of the 10th European Particle Accelerator Conference, Edinburgh, Scotland, 2006 (EPS-AG, Edinburgh, Scotland, 2006), p. 750.

[9] R. L. Geng et al., High gradient studies for ILC with single-cell re-entrant shape and elliptical shape cavities made of fine-grain and large-grain niobium, in Proceedings of the 22nd Particle Accelerator Conference, PAC-2007, Albuquerque, NM (IEEE, New York, 2007), p. 2337.

[10] R. L. Geng et al., World record accelerating gradient achieved in a superconducting niobium rf cavity, in Proceedings of the 21st Particle Accelerator Conference, Knoxville, TN, 2005 (IEEE, Piscataway, NJ, 2005), p. 653.

[11] R. L. Geng et al., Preliminary cryogenic cold test results of the first 9-cell LSF shape cavity, in Proceedings of the 
12th International Particle Accelerator Conference (IPAC'21), Campinas, Brazil, 2021 (JACoW Publishing, Geneva, 2021), pp. 2296-2299, 10.18429/JACoWIPAC2021-TUPAB342.

[12] V. Yakovlev et al., Progress towards of a superconducting traveling wave accelerating structure, Proceedings of the 22nd Particle Accelerator Conference, PAC-2007, Albuquerque, NM (Ref. [9]), p. 2182.

[13] H. Padamsee, ILC energy upgrade paths to $3 \mathrm{TeV}, 2021$ International Conference on $R F$ Superconductivity (SRF'21), WEPFAV006.

[14] A. Grassellino et al., Perspectives on superconducting linear colliders (ILC) to the next century. Part B: Energy upgrades to $3 \mathrm{TeV}$ and beyond, https://www.snowmass 21 .org.

[15] A. Kanareykin et al., An L-band superconducting traveling wave accelerating structure with feedback, AIP Conf. Proc. 1086, 445 (2008).

[16] V. Yakovlev et al., High-gradient tests of the single-cell SC cavity with the feedback waveguide, AIP Conf. Proc. 1299 , 313 (2010).

[17] R. Kostin, P. Avrakhov, A. Kanareykin, V. Yakovlev, and N. Solyak, Progress towards 3-cell superconducting traveling wave cavity cryogenic test, J. Phys. Conf. Ser. 941, 012100 (2017).
[18] D. G. Myakishev and V. P. Yakovlev, The new possibilities of SuperLANS code for evaluation of axisymmetric cavities, in Proceedings of the Particle Accelerator Conference, Dallas, TX, 1995 (IEEE, New York, 1995).

[19] C. Grimm (private communication).

[20] T. Higo, Progress of X-band accelerating structures, in Proceedings of the 25th International Linear Accelerator Conference, LINAC-2010, Tsukuba, Japan (KEK, Tsukuba, Japan, 2010).

[21] H. Padamsee, J. Knobloch, and T. Hays, RF Superconductivity for Accelerators (John Wiley, New York, 1998).

[22] V. Shemelin, H. Padamsee, and R. L. Geng, Optimal cells for TESLA accelerating structure, Nucl. Instrum. Methods Phys. Res., Sect. A 496, 1 (2003).

[23] V. Shemelin and S. Belomestnykh, Multipactor in Accelerating Cavities (Springer, Switzerland, 2020).

[24] S. Antipov, D. Sergatskov, and V. Yakovlev, Quench dynamics in SRF cavities, 2D Simulation of Quench Dynamics," Fermilab TD Technical Note TD-11-017, Fermilab, 2016.

[25] V. Shemelin, H. Padamsee, and V. Yakovlev, Optimization of a traveling wave superconducting radiofrequency cavity for upgrading the International Linear Collider, arXiv: 2105.12276. 